\title{
Introduction to the special issue on agent-based modelling for policy engineering
}

\author{
Francien Dechesne • Amineh Ghorbani • \\ Neil Yorke-Smith
}

Published online: 15 February 2014

(C) Springer-Verlag London 2014

\section{Introduction}

Should governments regulate financial markets more closely? Can investment on waste-based bio-gas systems improve farming prospects for animal farmers? How does taxation on light bulbs or subsidies on LED lamps influence the behaviour of consumers' energy usage habits? Does imposing fines on recycling companies in a developing country prevent them from hiring children and using dangerous chemicals when they are recycling electronic appliances? These questions all address policy problemsexploring the long-term effect of strategic decisions on the operational behaviour of individuals and on the global outcomes of a complex social system, such as a national economy.

The research of the first two authors received funding from the Dutch Next Generation Infrastructures Foundation's Empowering Networks program, under project numbers 09.04 and 09.08. The last author was supported in part by the University Research Board of the American University of Beirut under Grant number A88813.

\section{F. Dechesne - A. Ghorbani}

Department of Technology, Policy and Management, Delft

University of Technology, Jaffalaan 5, 2628 BX Delft,

The Netherlands

e-mail: f.dechesne@tudelft.nl

A. Ghorbani

e-mail: a.ghorbani@tudelft.nl

N. Yorke-Smith $(\square)$

Suliman S. Olayan School of Business, American University

of Beirut, Beirut, Lebanon

e-mail: nysmith@aub.edu.lb

N. Yorke-Smith

University of Cambridge, Cambridge, UK
Policies are rules or instruments that guide actions and decision-making by people in order to achieve certain desired outcomes in socio-technical systems. The design of effective policies is a cyclic process of identifying the objective, defining alternative policies, selecting those that best address the objective, and finally monitoring and evaluating the implemented policies' effects on the system (Weimer and Vining 2005). Throughout this cycle, various tools ranging from benchmarking and historical analysis (Scharpf 1997) to computational simulations (Gilbert and Troitzsch 2005) are used.

Whereas policies are implemented as top-down decisions, their acceptance is-at least partly-a bottom-up process. This calls for a system understanding at the microlevel in order to find out which of the alternative policies are most effective (Scharpf 1997). Micro-level analysis contributes insight into the individual's unanticipated adaptive behaviour, decision-making and interactions, facilitating the improvement of conditions for effective policy solutions.

The need for micro-level analysis fits with what agentbased modelling (ABM) offers. ABM, as a bottom-up simulation approach, builds artificial societies and abstractions of socio-technical systems, building them from individual agents and their interactions, and thus gives insight into how people may react towards different situations (Banks et al 2000). Compared with other computational approaches such as differential equations or statistical modelling, ABM imposes fewer assumptions on linearity, homogeneity, normality, and stationarity (Banks et al 2000). In addition, agent-based models have the power to demonstrate emergent phenomena at the system level. This is especially instrumental for policy problems where it is the influence of individual behaviour on system properties that is under study (Conteet al 2001). 
There are additional requirements in order to use ABM for policy analysis. For example, when evaluating policy alternatives, the policy analyst also needs means of imposing policies (or rules) to the simulated system in order to study individual reaction and adoption to these impositions. Therefore, building a system purely from the bottom upwards might not be entirely instrumental for policy analysis. Further, since the the subjects of policy problems are societies with real people, the reliability of an agent simulation and the results it provides are a delicate matter that requires careful evaluation.

\section{About the special issue}

This special issue is the outcome of the second workshop on agent-based modelling for policy engineering (AMPLE 2012), together with other papers holding the same focus.

The AMPLE workshop series has had instances in 2011 (co-located with the conference on autonomous agents and multi-agent systems, $A A M A S$ ), in 2012 (co-located with the European conference on artificial intelligence, ECAI, with a special track on Agent-Based Simulations for a Sustainable Society), and in 2013 as special track in the workshop on Coordination, Organization, Institutions and Norms in multi-agent systems, COIN (co-located with the conference on principles and practice of multi-agent systems, PRIMA).

The goal of the AMPLE workshop series is to bring together agent and artificial society research on the one hand, with policy analysis tools and policy analysts on the other hand. Bringing together different perspectives on similar problems problems help us see how agent research can be used and developed to assist policy making in business, governance, and society, and broadly in the social sciences.

The goal of this special issue is not only to gather examples of the requirements for and benefits of agentbased modelling as a policy analysis tool, but also to help identify the best practices for linking agent and artificial society research on the one hand, with policy making, institutional analysis, and tools such as system dynamics and gaming on the other. The combination could have benefits for the further enrichment of agent-based modelling and simulation as well as making ABM a increasingly useful tool for policy analysis. By gathering these different perspectives, we aim to explore how agent research can be used or improved to assist policy making in the social sciences.

The special issue editors express grateful thanks to the participants and reviewers of AMPLE'12 workshop, and to the reviewers of the articles in this special issue. We thank the AI \& Society editorial team and the Springer staff for their support. Special thanks are due to T. Savarimuthu.

\section{Contents}

The articles in this special issue cover a wide range of perspectives on the application of ABM for policy analysis. State-wide infrastructure projects are studied by Zia and Koliba. They ask how intergovernmental institutional rules, set by federal, state and regional government agencies, generate and sustain basins of attraction in funding infrastructure projects. Their article The Emergence of Attractors Under Multi-Level Institutional Designs: Agent Based Modeling of Intergovernmental Decision Making for Funding Transportation Projects develops a pattern-oriented ABM of an intergovernmental network in order to simulate real-world transportation policy implementation processes across the US federal, state of Vermont, regional, and local governments for prioritizing transportation projects.

Transportation policy is also the topic of Beheshti and Sukthankar's article. They study forecasting of road and parking usage, by means of a hybrid approach that combines agent-based and stochastic simulations in order to improve the accuracy and reduce the variance of long-term predictions. In their approach, an ABM is used to bootstrap the proposal distribution for the Markov Chain MonteCarlo estimator. Their article A Hybrid Modeling Approach for Parking and Traffic Prediction in Urban Simulations presents a case study in forecasting transportation patterns and parking lot utilization on a large university campus and shows significantly better predictions than other comparable modelling techniques.

Societal non-cooperation-where individuals have motivations for not cooperating with others-is addressed by Savarimuthu et al. A familiar example of non-cooperation is the issue of free-riding, where some agents do not contribute to the welfare of the society but do consume valuable resources. The article An Agent-based Simulation for Restricting Exploitation in Electronic Societies through Social Mechanisms simulates a decentralized peer-to-peer system which self-organizes itself to avoid cooperative sharers being exploited by uncooperative free riders. Specifically, the authors illustrate how cooperative sharers and uncooperative free riders can be placed in different groups of an electronic society in a decentralized manner. Thus, the article offers new insights into policy mechanisms for regulation of distributed societies.

A related social question is studied by Pitt, Busquets, and Riveret. In the setting of self-organizing autonomous agents of heterogenous provenance, they ask how the agents should distribute a collectivized set of resources amongst themselves. Is the allocation fair? Is the allocation method effective? Is it efficient? Are the decision makers accountable? The article The Pursuit of Computational Justice in Open Systems presents a formal model 
and some experimental results, and, concluding that the different aspects are all inter-connected, argues for a comprehensive research programme in computational justice.

Community-based allocation and coordination in energy distribution is an example of a situation demanding fairness and effectiveness. Yasir et al. present an agent-based architecture for coordinating locally connected microgrids, with the aim of supporting more cost-effective integration into the main power grid. Their article, Agentbased Community Coordination of Local Energy Distribution, shows through simulation experiments how the micro-grid agent society operates and adapts under varying conditions of renewable energy availability and energy demand patterns. The experimental design and evaluation are motivated by a policy modelling perspective in which the utility of an energy policy to a community is based on two attributes, the financial gain, and the reduction in carbon emissions.

Transition is also studied by Osinga, Kramer, and Hofstede in a different domain: animal welfare. In their article Sustainable Animal Welfare: Does Forcing Farmers into Transition Help?, Osinga et al. use ABM to investigate whether and how two different policies support the wellbeing of animal farmers when switching markets, and whether they benefit the society as a whole.
Finally, a broader issue is addressed by Jiang et al. who study the effect of institutional compliance on business processes and propose methods of automating regulatory control in organizations. The article Regulatory Compliance of Business Processes proposes a Consistency and Compliance Checker Framework to manage the complex interrelationships between organizational regulations. Through this framework, it can be checked whether the business processes comply with such regulations. Jiang et al. take a declaration management system as their case study to evaluate their proposed framework.

\section{References}

Banks J, Carson J, Nelson B, Nicol D (2000) Discrete-Event System Simulation, 3rd edn. Prentice-Hall, Upper Saddle River, New Jersey

Conte R, Edmonds B, Moss S, Sawyer R (2001) Sociology and social theory in agent based social simulation: a symposium. Comput Math Organ Theory 7(3):183-205

Gilbert G, Troitzsch K (2005) Simulation for the social scientist, 2nd edn. Open Univ Press, Milton Keynes, UK

Scharpf F (1997) Games real actors play: actor-centered institutionalism in policy research. Westview Press, Boulder, Colorado

Weimer D, Vining A (2005) Policy analysis: Concepts and practice, 4th edn. Prentice Hall, Upper Saddle River, New Jersey 